\title{
SGB II-Bildungsberater gesucht
}

\section{Die berufliche Bildungsberatung kommt in den Jobcentern zu kurz}

\author{
KLAUS KEMPKENS \\ Dr. Klaus Kempkens ist Wissen- \\ schaftliche Lehrkraft für Beratung \\ und Vermittlung an der Hochschule \\ der Bundesagentur für Arbeit in \\ Schwerin. Zuvor war er 24 Jahre als \\ Beratungs- und Führungskraft für \\ SGB II und III in der Bundesagentur \\ für Arbeit tätig \\ klaus.kempkens@arbeitsagentur.de
}

\author{
Die vermittlerischen und beraterischen Aufgaben \\ in den Arbeitsagenturen des Rechtskreises SGB III \\ wurden bis ins Jahr 2004 von zwei Fachkräftegruppen \\ durchgeführt, den Arbeitsvermittlern und \\ den Arbeitsberatern. Das Tätigkeitsprofil der \\ Arbeitsberater wurde 2004 in der Bundesagentur \\ flächendeckend abgeschafft. Deren Aufgaben werden \\ seitdem von Arbeitsvermittlern übernommen.
}

Mit Einführung des SGB II (»Hartz $I V «)$ zum 1. Januar 2005 erfolgte eine Aufteilung der Kundengruppen in SGB II (Grundsicherung) und SGB III (Versicherungsleistung). Im SGB III liegt seither das Primat auf Vermittlung in Arbeit, so dass Beratungen im Kontext beruflicher Bildung in den Hintergrund treten. Falls sich in Einzelfällen doch Bedarf an beruflicher Beratung zeigt, so wird diese Aufgabe nunmehr seitens der Arbeitsvermittler en passant erledigt.

In der SGB II-Grundsicherung hat man sich der Struktur des seit 2004 verschlankten SGB III-Bereichs angepasst. Seit 2005, mit Umsetzung des neuen SGB II-Gesetzes, sind, analog zum SGB III, ausschließlich Arbeitsvermittler oder »Persönliche Ansprechpartner $(\mathrm{PAP})$ « im SGB II beraterisch sowie vermittlerisch in Personalunion tätig. Eine Ausnahme ist das Fallmanagement für Leistungsempfänger mit multiplen Vermittlungshemmnissen. Es wurde darauf verzichtet, im SGB II entsprechende Beratungsfachkräfte zu installieren (z. B. Arbeitsberater klassischer Prägung oder eine Berufsbildungsberatung). Im Folgenden soll gezeigt werden, dass der Bedarf an Beratung zu Fragen beruflicher Bildung sehr hoch ist und nach Einschätzung des Verfassers sogar größer ist als er im Rechtskreis SGB III jemals war.

\section{Aufgabenzuschnitt ehemaliger Arbeitsberater}

Die Aufgabe der Arbeitsberater bestand in der Beratung arbeitsloser weiterbil- dungswilliger Menschen über 25 Jahren. Für den Jugendbereich (bis 25 Jahre) waren und sind bis zum heutigen Tage Berufsberater (U25 SGB III) zuständig. Beide Beratergruppen boten und bieten in dem komplexen und fluidem Feld der Berufsbildung sowohl Orientierung, Beratung als auch die Teilnahme an berufsbildenden Maßnahmen (zum Teil mit der Möglichkeit, formale Zertifikate wie Berufsabschlüsse zu erwerben).

Arbeitsberater waren Experten zu Themen beruflicher (Weiter-) Bildung Erwachsener und bewegten sich im Themendreieck von Individuum, Bildungssystem und Arbeitsmarkt. Sie verfügten über hohe Kompetenz im Kontext der Feststellung individueller beruflicher Eignung und Neigung sowie über profunde berufskundliche Kenntnisse. Sie hatten einen Überblick über den regionalen Angebots- und Nachfragemarkt für Arbeitskräfte und zugleich zukünftige regionale Entwicklungen im Visier, um zyklische Qualifizierungen (in Absprache mit lokalen Bildungsdienstleistern) zu initiieren, um die benötigten Facharbeitskräfte zu generieren.

Auf dieser Basis war es ihnen möglich, passende Angebote für die individuellen Wünsche und Bedürfnisse der arbeitslosen Kunden zu finden. Der ganzheitliche Ansatz sah vor, dass Arbeitsberater neben der Beratung auch die individuellen Kostenzusagen für ausgewählte Bildungsziele erteilten (Bildungsgutscheine). Eine Umschulung kann bis zu 28 Monate (für dreieinhalbjährige Ausbildungen unter 
Berücksichtigung des Verkürzungspostulats um ein Drittel der regulären Ausbildungszeit gemäß $\$ 180$ Abs. 4 SGB III) dauern und gerade in technischen Berufen sind recht hohe Monatsteilnehmerpauschalen Usus (bis ca. 1.400 Euro).

Somit sind Kosten bis zu 35.000 Euro für einen Umschulungsförderungsfall einzuplanen, so dass etwaige Fehlallokationen für die Organisation (seit 2005 lokales SGB II-Jobcenter bzw. örtliche Agentur für Arbeit bis 2004) kostspielig und für den Kunden demotivierend sind sowie im Falle eines Abbruchs eine nicht beabsichtigte berufliche Sackgasse darstellen (vgl. »misleading trajectories", Anm. 1). Berufliche Beratung, Eignungsabklärung, Kenntnisse und Erfahrungen auf dem regionalen Arbeitsmarkt sowie Erkennen von Entwicklungen waren zentrale Aufgaben zur Vermeidung von Fehlallokationen und um die eingesetzten finanziellen Mittel gezielt, sachgerecht, erfolgreich einsetzen zu können.

Der Weg, um in der Arbeitsberatung tätig sein zu können, führte seinerzeit über eine sechsmonatige Vollzeit-Weiterbildung im Anschluss an eine langjährige überdurchschnittlich bewertete Tätigkeit als Arbeitsvermittler oder über eine spezialisierte akademische Ausbildung an der Hochschule der Bundesagentur für Arbeit (HdBA, bis 2006 Fachhochschule des Bundes, FB Arbeitsverwaltung). Diese Wege in die Arbeitsberatung indizieren, dass es sich um einen hochwertigen und anspruchsvollen Aufgabenzuschnitt gehandelt hat.

\section{Hoher Bedarf nach Bildungsberatung in den SGB II-Jobcentern}

Vor der Einführung von Hartz IV war im Rechtskreis SGB III die Kombination von Arbeits-vermittlung (AV) und Arbeitsberatung (AB) sinnvoll und hatte sich über die Jahre bewährt, da der eine besonders kompetent für konkrete Vermittlung in Arbeit und der andere Spezialist für berufliche Beratung Erwachsener über 25 Jahren war. Für die Tätigkeit eines Arbeitsberaters bestand jedoch seit 2005 mit Einführung des SGB II (Hartz IV) kein Bedarf mehr. Der Arbeitslosengeld I-Bereich (SGB III) zog sich auf die klassische Arbeitsvermittlung zurück, da zum großen Teil KurzzeitArbeitslose (die meisten mit beruflichem Abschluss) betreut werden, bei denen es vorrangig um die Wiederaufnahme eines versicherungspflichtigen Beschäftigungsverhältnisses geht. Falls eine Vermittlung in Arbeit innerhalb des ALG I-Gewährungszeitraums nicht gelingen sollte, erfolgt zumeist eine Überstellung an die Grundsicherung des SGB II.

Ein Blick auf die Struktur der Leistungsbezieher im Rechtskreis SGB II zeigt, dass bundesweit mehr als die Hälfte der Arbeitslosen über keinen eigenen beruflichen Abschluss verfügt (55\%, Anm. 2). In den westdeutschen Großstädten liegt die Quote der Geringqualifizierten sogar bei 63 bis $70 \%$ (3). Der Hartz IV-Bereich stellt somit ein Sammelbecken für Leistungsempfänger mit dringendem Qualifizierungsbedarf dar.

Insgesamt sind 1,3 Millionen (4) formal nicht qualifizierte Menschen offiziell arbeitslos gemeldet, der überwiegende Teil ist mit 1,1 Millionen dem Rechtskreis SGB II (Hartz IV) zugeordnet. Besonderer Handlungsbedarf besteht bei den jungen Erwachsenen zwischen 25 und 35 Jahren. Der klassische (duale) Ausbildungszug ist für diese in fast allen Fällen abgefahren, dennoch steht beispielsweise ein 25-Jähriger dem Arbeitsmarkt immerhin noch 42 Jahre lang zur Verfügung (falls im Ungelernten-Status verbleibend entweder als Hilfskräfte in prekärer Beschäftigung oder temporär als Arbeitslose).

Wie akut der bestehende Handlungsbedarf ist, zeigt eine Untersuchung im Bundesland Hessen. Zwei Drittel (67\%, Anm. 5) der 25 bis35-jährigen Hartz IV-Arbeitslosen verfügen dort über keine Berufsausbildung. Trotz eines zukünftig steigenden Fachkräftebedarfs können sich diese auf dem Arbeitsmarkt lediglich als Hilfskräfte positionieren, wobei der Bedarf an ungelernten Arbeitskräften im Trend (6) zurück-geht.

In einem im Oktober 2013 veröffentlichten Beitrag konstatiert das Institut für Arbeitsmarkt- und Berufsforschung, dass eine berufliche Ausbildung der beste Schutz vor Arbeitslosigkeit ist. Während die Arbeitslosenquote für Menschen mit Berufsabschluss in Deutschland bei 5 \% liegt, liegt die Quote nicht formal Qualifizierter bei $19 \%$ (7), also fast viermal so hoch.

Bundesregierung als auch Bundesagentur für Arbeit sehen Handlungsbedarf und haben kürzlich mit entsprechenden Initiativen reagiert. Die Bundesagentur trägt der Bedeutung fehlender Berufsabschlüsse insbesondere junger Erwachsener
Rechnung und hat für 2013 als wichtigstes geschäftspolitisches Handlungsfeld in der Grundsicherung das Ziel formuliert, »Kunden ohne Abschluss zu Fachkräften aus(zu)bilden« (8) (Planungsbrief SGB II). Die Kampagne des Bundes »Ausbildung wird was - Spätstarter gesucht " (9) in Verbindung mit der Initiative der Bundesagentur »Erstausbildung junger Erwachsener « (10) geben den Startschuss für eine mehrjährige berufsabschlussorientierte Bildungsoffensive. Im Fokus steht hierbei die Nutzung nicht besetzter Ausbildungsstellen des dualen Ausbildungsmarktes. Zuzüglich sollen weitere Schwerpunkte auf betrieblichen oder trägerorientierten klassischen Umschulungen $(\mathbb{S} \mathbb{S} 81 \mathrm{ff}$. SGB III) sowie zielgruppenspezifisch angepassten modularen Konzepten liegen.

\section{Beratungslücke in den Jobcentern}

Die einzige operativ zur Verfügung stehende Mitarbeitergruppe, die Arbeitsvermittler, haben bereits ein sehr breites Aufgabenspektrum. Die berufliche Bildungsberatung ist nur eine dieser Aufgaben und wird nebenbei mit erledigt. Erschwerend kommt hinzu, dass Arbeitsvermittler noch nicht einmal ansatzweise hierfür ausgebildet sind (gemessen am Kompetenzprofil und Ausbildungsweg der ehemaligen SGB III-Arbeitsberater). Wenn die erwähnte Berufsabschluss-Offensive von Bundesarbeitsministerium und Bundesagentur anläuft, wird hierdurch ein noch größerer Beratungsbedarf generiert. Diese Aufgabe ist seitens der Arbeitsvermittler in sachgerechter und erfolgversprechender Weise kaum zu schultern.

Neben der klassischen Arbeitsvermittlung, der Fülle zu gewährender finanzieller Eingliederungsleistungen aus SGB II und III gehört es zusätzlich den Aufgaben eines Arbeitsvermittlers, ohne spezielle Schulungen und vielfach ohne adäquate Kenntnisse, profunde Bildungsberatungen (insbesondere Umschulungen) durchzuführen. Diese sind rechtlich und finanziell verbindlich, $\mathrm{da}$ zur Beratungsleistung auch Erstellung von Kostenzusagen für avisierte Bildungsziele (Bildungsgutscheine) gehört.

In der Regel fehlt den Arbeitsvermittlern das erforderliche Handwerkszeug; viele haben als ab 2005 eingestellte externe Mitarbeiter lediglich ein kurzes On-The-Job-Training mit dem Fokus auf Arbeitsvermittlung erhalten. Zu den 
Themenkreisen Berufskunde (zurzeit 330 duale Ausbildungsberufe sowie länderspezifische schulische Ausbildungen), Cluster von Berufen, Anforderungen in Berufen, Feststellung von Eignung und Neigung, alternative Berufswegplanungen, Weiterbildungssysteme, Arbeitsmarktkunde, allgemeine Bildungssysteme im Bund und dem jeweiligen Bundesland, spezielle Beratungskompetenzen, verfügen Arbeitsvermittler in der Regel über kaum mehr als allgemeines Jedermann-Wissen und sind aufgrund von Wissensdefiziten kaum in der Lage, professionell und zielgruppenadäquat zu beraten. So kommt es häufig zu individuell suboptimalen Beratungsergebnissen. Zusammenfassend ist zu konstatieren, dass Arbeitsvermittler in der Regel nicht nur hinsichtlich der Breite ihres Aufgabenspektrums, sondern auch fachlich häufig überfordert sind.

\section{Beratungsauftrag und Beratung als Kernkompetenz}

Der politische Wille zu Optimierung der beruflichen Bildung gerade für Kunden der Grundsicherung sowie die damit verbundene Erhöhung des Stellenwertes von Beratung zeigt sich eben den genannten flächendeckenden Offensiven auch in den Vereinbarungen der neuen schwarz-roten Bundesregierung von Dezember 2013.

Ein Blick in den neuen Koalitionsvertrag (S. 30-38, Anm. 11) bestätigt den eingeschlagenen Weg. Die neue Bundesregierung will »einen Schwerpunkt auf die Stärkung der beruflichen Bildung legen « und wird eine »Allianz für Aus- und Weiterbildung « ausrufen und. In diesem Kontext sollen »Weiterbildungsinteressierte systematisch beraten werden «. Im weiteren Verlauf der Vereinbarung werden einzelne, typische Hartz IV-Zielgruppen genannt wie Geringqualifizierte, Langzeitarbeitslose, Alleinerziehende sowie Arbeitslose mit Migrationshintergrund, die einer »fachgerechten Beratung « bedürfen. Insbesondere den Geringqualifizierten sowie Langzeitarbeitslosen sollen »neue Chancen erschlossen werden «. Dieses ist nicht ohne fachgerechte und intensive Beratung zu bewerkstelligen.

Wenn nun abschlussorientierte Bildung oben auf der politischen Agenda steht, erscheint es sinnvoll, wenn nicht sogar geboten, (Berufsbildungs-) Beratungsexperten in den SGB II-Jobcentern einzusetzen. Es wäre zumindest einen Versuch wert, beispielsweise als Probelauf im Zeitraum der gerade gestarteten dreijährigen Bildungsoffensive hinsichtlich der Berufsabschlüsse junger Erwachsener.

Eine der Kernkompetenzen, welche die Bundesagentur für Arbeit traditionell für sich reklamiert, ist ihre Beratungskompetenz, die auch in den Vorschrif-

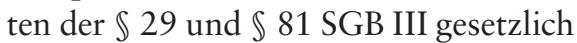
verankert ist. Die Bundesagentur ist an den Nahtstellen von Schule und Beruf (Berufsberater), Arbeitslosen und Arbeitsmarkt (Arbeitsvermittler SGB II und III), Deckung des Fachkräftebedarfs durch Schaffung von Fachkräften durch abschlussorientierte Weiterbildung (derzeit Arbeitsvermittler) beraterisch tätig. Neben dem direkten Zugang zu den Kunden verfügt die Bundesagentur über die finanziellen Mittel (Eingliederungstitel der Jobcenter) Arbeitsmarktpolitik vor Ort als auch flächendeckend zu gestalten, um ihre gesetzlichen Aufträge zu erfüllen.

Ihre Aufgaben sind es unter anderem, den »Ausgleich von Angebot und Nachfrage auf dem Ausbildungs- und Arbeitsmarkt « zu unterstützen. "Dabei ist insbesondere durch die Verbesserung der individuellen Beschäftigungsfähigkeit Langzeitarbeitslosigkeit zu vermeiden«( $\$ 1$ Abs. 1 SGB III). Die Förderung individueller Beschäftigungsfähigkeit bedeutet, den "Erhalt und Ausbau von Fertigkeiten, Kenntnissen und Fähigkeiten « zu fördern ( $\mathbb{1} 1$ Abs. 2 Nr. 2 SGB III). Bei den in dieser Rechtsnorm speziell angesprochenen Langzeitarbeitslosen, die überwiegend über keinen beruflichen Abschluss verfügen, geht es in vielen Fällen um den nachqualifizierenden Erwerb eines solchen Abschlusses.

Die Erfüllung ihrer unmittelbaren Beratungsaufgaben und Beratung als conditio sine qua non insbesondere zur Vorbereitung individuell-passgenauer Weiterbildungen von gering qualifizierten Hartz IV-Kunden erscheint ohne speziell ausgebildete Beratungsfachkräfte fachlich kaum zu bewerkstelligen. Neu geschaffene Berufsbildungsberater könnten diese Schlüsselaufgaben von den Allroundern, den Arbeitsvermittlern, übernehmen. Diese wiederum besitzen dann mehr Zeit für ihre eigentlichen Kernaufgaben, die Vermittlung von SGB II-Kunden in Arbeit.

\section{Einführung einer Hartz IV- Berufsbildungsberatung}

Mit der Einführung einer Berufsbildungsberatung (BBB) würden Kosten für defi- zitäre Beratungen und Fehlallokationen finanzieller Mittel (Eingliederungstitel des jeweiligen Jobcenters) reduziert und idealerweise weitgehend vermieden. In Zeiten reduzierter Jobcenter-Eingliederungsbudgets erscheint eine effiziente und erfolgreiche Nutzung der verfügbaren Mittel umso mehr geboten. Die Gesamtkosten der aktiven Arbeitsmarktförderung im Rechtskreis SGB II betrugen 2,3 Milliarden Euro im Jahr 2012. Insgesamt 654 Millionen (12) Euro, das sind $28 \%$, wurden im Rechtskreis SGB II für berufliche Bildung ausgegeben (ohne zugelassene kommunale Träger). Zum jetzigen Zeitpunkt (Oktober 2013) befinden sich 158.000 Kunden des Rechtskreises SGB II in Maßnahmen der beruflichen Weiterbildung, davon erwerben 61.500 einen beruflichen Abschluss (13).

Falls die neue Bundesregierung ihre im Koalitionsvertrag niedergelegte Absichtserklärung, den Fokus verstärkt auf Bildung zu legen, umsetzt und Berufsabschlüsse für arbeitslose junge Erwachsene besonders fördert, besteht bereits in naher Zukunft ein erhöhter diesbezüglicher Beratungsbedarf. Die Arbeit an der Basis wird durch die Fachkräfte der Jobcenter übernommen (individuelle Beratungen, Festlegung der Bildungsziele und Bindung der finanziellen Mittel). Es obliegt der Bundesagentur für Arbeit, diese wichtige Beratungsarbeit, wie bisher nebenbei von nicht speziell ausgebildeten Arbeitsvermittlern erledigen zu lassen oder wie skizziert qualifizierte Berufsbildungsberater einzusetzen. Der Einsatz solcher Beratungsfachkräfte ist aus fachlicher wie auch finanzieller Sicht sinnvoll.

Das Plädoyer hinsichtlich der Einführung einer Berufsbildungsberatung passt in die gegenwärtige Zielausrichtung der Bundesagentur für Arbeit, die derzeit eine Professionalisierungs-Strategie einiger Fachkräftegruppen und im SGB III sogar die Neuinstallation einer Fachkraftgruppe betreibt. Das Projekt »Interne ganzheitliche Integrationsberatung « wird institutionalisiert und sogenannte Integrationsberater SGB III (für Kunden mit komplexen Problemlagen) installiert. Darüber hinaus wird auch für den SGB II-Bereich eine Professionalisierung der Fallmanager (zuständig für Arbeitslose mit multiplen Vermittlungshemmnissen) angestrebt. Neue und auch bereits eingesetzte Fallmanager werden nach DGCC18-Richtlinien geschult und erwerben eine anerkannte Zertifizierung. 
Wenn auch weiterhin dem Bedarf nach zielgruppengenauer Kundenbetreuung in SGB II sowie III Rechnung getragen wird, dann passen sowohl das Plädoyer nach Einführung von Beratungsspezialisten für berufliche Bildung als auch die erforderlichen Schulungen in den Gesamtkontext einer Professionalisierung der operativen Fachkräfte der Bundesanstalt für Arbeit. Die Bundesagentur für Arbeit hat im letzten Herbst eine großangelegte Medienkampagne unter dem Motto gestartet »Wenn nicht jetzt- wann dann «. Dies gilt auch für die Schließung der Bildungs-Beratungslücke im Bereich Hartz IV.

\section{Anmerkungen}

(1) Institute for Regional Innovation and Social Research (TSER/IRIS) (2003): Misleading Tractories? An Evaluation of the Unintended Effects of Labour Market Integration Policies for Young Adults in Europe, Final Report, April 2001 (updated Jan. 2013). http:// cordis.europa.eu/documents/ documentlibrary/70601461EN6. pdf Stand 20.11.2013

(2) Bundesagentur für Arbeit (2013): Grundsicherung für Arbeitsuchende in Zahlen, Oktober 2013, Tabelle 3.7.

(3) Klaus Kempkens (2011): Berufspädagogische Untersuchung zur Hartz IV-Arbeitsmarktförderung, Kovac, Hamburg.

(4) Bundesagentur für Arbeit (2013a): Arbeitslose nach Rechtskreisen, Oktober 2013.

(5) Regionaldirektion Hessen der Bundesagentur für Arbeit (2013): Sozialgesetzbuch II /Entwicklungen in Hessen 2012.

(6) Bundesagentur für Arbeit (2013a): Arbeitslose nach Rechtskreisen, Oktober 2013 sowie Institut für Arbeitsmarkt- und Berufsforschung/ IAB (2013): Qualifikationsspezifische Arbeitslosenquoten, Aktuelle Daten und Indikatoren, Nürnberg, 14.10.2013.

(7) Institut für Arbeitsmarkt- und Berufsforschung /IAB (2013): a. a. O.

(8) Bundesagentur für Arbeit (2012): Planung und Steuerung 2013 für die gemeinsamen Einrichtungen der Grundsicherung, Nürnberg, 19.11.2012.
(9) Bundesministerium für Arbeit und Soziales (2013): Kampagne »Ausbildung wird was - Spätstarter gesucht «, Berlin.

(10) Bundesagentur für Arbeit (2013b): Erstausbildung für junge Erwachsene, Eine Initiative der Agenturen für Arbeit und Jobcenter, Nürnberg.

(11) Koalitionsvertrag CDU-CSU-SPD, Dezember 2013, 18. Legislaturperiode, Berlin.

(12) Bundesagentur für Arbeit (2012a): Förderstatistik/Daten zu den Eingliederungsbilanzen, Jahresabschluss 2012, Tabelle 2.

(13) Bundesagentur für Arbeit (2013e): Arbeitsmarkt in Zahlen, Förderstatistik der BA, Datenstände September/Oktober 2013.

\section{Literatur}

Bundesagentur für Arbeit (2012): Planung und Steuerung 2013 für die gemeinsamen Einrichtungen der Grundsicherung, Nürnberg, 19.11.2012.

Bundesagentur für Arbeit (2012a): Förderstatistik/Daten zu den Eingliederungsbilanzen, Jahresabschluss 2012, Tabelle 2.

Bundesagentur für Arbeit (2013): Grundsicherung für Arbeitsuchende in Zahlen, Oktober 2013. Bundesagentur für Arbeit (2013a): Arbeitslose nach Rechtskreisen, Oktober 2013

Bundesagentur für Arbeit (2013b): Erstausbildung für junge Erwachsene, Eine Initiative der Agenturen für Arbeit und Jobcenter, Nürnberg. Bundesagentur für Arbeit (2013C): Jüngere

Menschen ohne Berufsabschluss, Der Arbeitsmarkt in Deutschland, Mai 2013

Bundesagentur für Arbeit (2013d): Tarifvertrag der BA, Anlagen 3 und 9 zum 11. Änderungs-TV zum TV-BA, Zuordnungstabelle für den Rechtskreis SGB II (gE)/Gehaltstabelle.

Bundesagentur für Arbeit (2013e): Arbeits-

markt in Zahlen, Förderstatistik der BA

Datenstände September/Oktober 2013.

Bundesagentur für Arbeit (2013f): HEGA 11/13-09 Bundesagentur für Arbeit (2013g): Wenn nicht jetzt- wann dann, Dialog Extra, 2/2013.

\section{Bundesministerium für Arbeit und Soziales}

(2013): Kampagne "Ausbildung wird was -

Spätstarter gesucht«, Berlin

CDU-CSU-SPD (2013): Deutschlands Zukunft gestalten, Koalitionsvertrag 2013, 18. Legislaturperiode, Berlin.

Institute for Regional Innovation and Social Research (TSER/IRIS) (2003): Misleading

Tractories? An Evaluation of the Unintended Effects of Labour Market Integration Policies for Young Adults in Europe, Final Report, April 2001 (updated Jan. 2013). http://cordis.europa. eu/documents/documentlibrary/70601461EN6. pdf. Stand 20.11.2013

Institut für Arbeitsmarkt- und Berufsforschung/IAB (2012): Der Einstieg in den Arbeitsmarkt prägt den Erwerbsverlauf, Presseinformation vom 17.10.2012

Institut für Arbeitsmarkt- und Berufsforschung /IAB (2013): Qualifikationsspezifische Arbeitslosenquoten, Aktuelle Daten und Indikatoren, Nürnberg, 14.10.2013.

Kempkens, K. (2011): Berufspädagogische Untersuchung zur Hartz IV-Arbeitsmarktförderung, Kovac, Hamburg.

Regionaldirektion Hessen der Bundesagentur für Arbeit (2013): Sozialgesetzbuch II /Entwicklungen in Hessen 2012.

Sozialgesetzbuch (2011/2013): Zweites und Drittes Buch (SGB II und III), Berlin, Fassung vom 13.05.2011 (SGB II) und 17.06.2013 (SGB III).



StichwortKommentar Behindertenrecht Herausgegeben von Prof. Dr. Olaf Deinert und Prof. Dr. Felix Welti 2014, ca. 1.100 S., geb., ca. 98,-€ ISBN 978-3-8329-7326-1 Erscheint ca. April 2014

Der Stichwortkommentar definiert alphabetisch konkrete Lebens- und Rechtsfragen, vertieft sie im juristischen Kontext und zeigt Lösungen für typische Beratungs- und Entscheidungssituationen auf.

www.nomos-shop.de/14506



